\title{
Ulam stability results for the solutions of nonlinear implicit fractional order differential equations
}

\author{
Zeeshan Ali*1 (D), Akbar Zada $^{1}$ (D), Kamal Shah² (iD \\ ${ }^{1}$ Department of Mathematics, University of Peshawar, Peshawar, Khyber Pakhtunkhwa, Pakistan \\ ${ }^{2}$ Department of Mathematics, University of Malakand, Dir(L), Khyber Pakhtunkhwa, Pakistan
}

\begin{abstract}
In this manuscript, we study the existence and uniqueness of solution for a class of fractional order boundary value problem (FBVP) for implicit fractional differential equations with Riemann-Liouville derivative. Furthermore, we investigate different kinds of Ulam stability such as Ulam-Hyers stability, generalized Ulam-Hyers stability, Ulam-HyersRassias stability and generalized Ulam-Hyers-Rassias stability for the proposed problem. The concerned analysis is carried out through using classical technique of nonlinear functional analysis. The main results are illustrated by providing a couple of examples
\end{abstract}

Mathematics Subject Classification (2010). 26A33, 34B27, 39B82

Keywords. Riemann-Liouville fractional derivative, implicit fractional differential equations, existence, Green function, Ulam-Hyers stability

\section{Introduction}

Fractional order differential equation is a generalization of the integer order differential equation. The idea of fractional calculus has been introduced at the end of sixteenth century (1695), when Leibnitz introduced the symbol $\frac{d^{p}}{d t^{p}} f(t)$ to denote the $p^{\text {th }}$ order derivative of a function $f$. L' Hospital wrote a letter to Leibnitz, in which he asked a question about the derivative of order $p=\frac{1}{2}$. This question was the foundation of the recent fractional calculus. Later on the fractional derivative was introduced by Lacroix [23]. Moreover, geometrical and physical interpretation for aforementioned area was also a very big problem for more than 300 years. Because there were not admissible interpretation for fractional order derivative and integration, like for integer order. In [28], it is evident that the geometric interpretation of fractional integration is "Shadows on the walls" and its physical interpretation is "Shadows of the past".

In the past few decades, fractional calculus has got incredible attention from the researchers. Fractional differential equations have been proved to be strong tools in the modeling of many physical phenomena. It is because, fractional order models are more accurate than integer order models as fractional order models allow more degrees of freedom.

\footnotetext{
*Corresponding Author.

Email addresses: zeeshanmaths1@gmail.com (Z. Ali), zadababo@yahoo.com (A. Zada), kamalshah408@gmail.com (K. Shah)

Received: 10.08.2017; Accepted: 09.02.2018
} 
The aforesaid equations have widespread applications in many areas of science and technology. Areas which utilized fractional differential equations include diffusion process [24], electrochemistry [26], biology [31], signal and image processing [33], process of dynamics [40], systems control theory [42] etc. For more applications of aforementioned equations, see $[5,6,12,19,27,28]$ and references therein.

One of the most preferable research area in the field of fractional order differential equation, which has got incredible attention from the researchers is devoted to the existence theory of solutions. This is an expeditiously growing area for analysis. For detail study about the existence theory, see $[2,8,10,18,34-37]$ and references therein. Since most of the engineering, physical and dynamical problems are subjected to the boundary conditions. Therefore researchers have given much attention to the study of considered area: Authors in [38], investigated a class of nonlinear FBVP of Dirichlet-type given by

$$
\begin{gathered}
D^{p} u(t)=-f(t, u(t)), t \in(0,1), p \in(1,2], \\
u(0)=u(1)=0,
\end{gathered}
$$

where $D^{p}$ stands for standard Riemann-Liouville derivative of fractional order and the function $f:[0,1] \times \mathbb{R}^{+} \cup\{0\} \rightarrow \mathbb{R}^{+} \cup\{0\}$ is continuous. Ahmad and Nieto [1], studied the existence and uniqueness of the following nonlinear boundary value problem by using Leray-Schauder fixed point theorem

$$
\begin{aligned}
D^{p} u(t)= & f(t, u(t)), t \in[0, T], T>0, p \in(1,2], \\
D^{p-2} u\left(0^{+}\right) & =\gamma D^{p-2} u\left(T^{-}\right), \\
D^{p-1} u\left(0^{+}\right) & =\beta D^{p-1} u\left(T^{-}\right),
\end{aligned}
$$

where the function $f:[0,1] \times \mathbb{R} \times \mathbb{R} \rightarrow \mathbb{R}$ is continuous and $\beta, \gamma \neq 1$.

Since, in most of the situations to find the exact solutions of nonlinear problems is quite difficult task. Therefore, different approximation techniques were developed to find numerical solution. From the numerical and optimization point of view, stability is very important. So various kinds of stability have been investigated such as Exponential, Lyapunov and Asymptotic stability etc. Stability is a very prominent branch of the qualitative theory of differential equations. Here in this manuscript, we will discuss Ulam-Hyers stability. The mentioned stability was first pointed out by Ulam [41], in 1940. In the following year, Hyers [13] gave a partial favorable answer to the question of Ulam in the context of Banach spaces. Furthermore, Rassias [29] improved the answer of Hyers. Later on the aforesaid stability was greatly discussed for functional equations (see [14,30]). Latterly, the results were generalized and extended by many researchers, readers may see $[17,20-22,25,39,45-47]$ and references therein. The mentioned stability is rarely studied for fractional differential equations and especially for FBVPs. Very few papers in this region can be found in literature, see $[3,4,7,16,43,44]$ and references therein.

Influenced from the aforesaid discussion, in this manuscript, we are investigating the existence, uniqueness as well as four types of Ulam stability for the considered FBVP of implicit fractional order differential equation. The proposed implicit boundary value problem, involving Riemann-Liouville fractional derivative, is given by

$$
D^{p} u(t)=f\left(t, u(t), D^{p} u(t)\right), t \in \mathcal{J}=[0, T], T>0, p \in(1,2],
$$

with the boundary conditions of fractional order

$$
\begin{aligned}
& D^{p-2} u\left(0^{+}\right)=\gamma D^{p-2} u\left(T^{-}\right), \\
& D^{p-1} u\left(0^{+}\right)=\beta D^{p-1} u\left(T^{-}\right),
\end{aligned}
$$

where $\beta, \gamma \neq 1$. We establish some adequate conditions for the existence and uniqueness of solutions to the given FBVP (1.1) - (1.3) through using fixed point theorems of Schaefer and Banach contraction type. Then by using standard functional analysis technique, we 
will establish applicable results under which the solution of the considered boundary value problem satisfies the conditions of different kinds of Ulam stabilities. The established results are demonstrated by couple of suitable examples.

\section{Background materials and auxiliary results}

In this portion, we recall some basic definitions, notations and preliminary results, which will be used throughout in the manuscript.

Definition 2.1. [1] The Riemann-Liouville fractional integral of order $p>0$ for a continuous function $u: \mathbb{R}^{+} \rightarrow \mathbb{R}$ is defined as

$$
I^{p} u(t)=\frac{1}{\Gamma(p)} \int_{0}^{t}(t-\zeta)^{p-1} u(\zeta) d \zeta
$$

provided that integral on right is pointwise defined on $(0, \infty)$. Here

$$
\Gamma(p)=\int_{0}^{\infty} t^{p-1} e^{-t} d t, p>0 .
$$

Definition 2.2. For a function $u: \mathbb{R}^{+} \rightarrow \mathbb{R}$, the Riemann-Liouville derivative of fractional order $p>0, n=[p]+1$ is defined as

$$
D^{p} u(t)=\frac{1}{\Gamma(n-p)}\left(\frac{d}{d t}\right)^{n} \int_{0}^{t}(t-\zeta)^{n-p-1} u(\zeta) d \zeta=\left(\frac{d}{d t}\right)^{n} I^{n-p} u(t),
$$

provided it exists. Here $[p]$ denotes the integer part of the real number $p$. For more properties of Riemann-Liouville derivative, reader may see [1]

Lemma 2.3. [1] The solution of the differential equation

$$
D^{p} u(t)=\theta(t), p>0,
$$

will be in the following form

$$
I^{p} D^{p} u(t)=I^{p} \theta(t)+k_{1} t^{p-1}+k_{2} t^{p-2}+\cdots+k_{n-1} t^{p-n-1}+k_{n} t^{p-n},
$$

where $k_{i}, i=1,2, \ldots, n$, are arbitrary real constants and $n=[p]+1$.

Lemma 2.4. The space $X=C^{1}(\mathcal{J}, \mathbb{R})$, is a Banach space endowed with a norm, defined by

$$
\|u\|_{X}=\max _{t \in \mathcal{J}}\{|u(t)|: t \in \mathcal{J}\} .
$$

Definition 2.5. [32] The FBVP (1.1)-(1.3) is said to be Ulam-Hyers stable if there exists $\mathcal{K}_{0} \in \mathbb{R}^{+}$such that for every $\epsilon>0$ and for every solution $u \in C^{1}(\mathcal{J}, \mathbb{R})$ of the inequality

$$
\left|D^{p} u(t)-f\left(t, u(t), D^{p} u(t)\right)\right| \leq \epsilon, t \in \mathcal{J},
$$

there exists a unique solution $v \in C^{1}(\mathcal{J}, \mathbb{R})$ of the considered problem (1.1)-(1.3),such that

$$
|u(t)-v(t)| \leq \mathcal{K}_{0} \epsilon, t \in \mathcal{J} .
$$

Definition 2.6. [32] The FBVP (1.1)-(1.3) is said to be generalized Ulam-Hyers stable if there exists $\varphi \in C\left(\mathbb{R}^{+}, \mathbb{R}^{+}\right), \varphi(0)=0$, such that for every solution $u \in C^{1}\left(\mathcal{J}, \mathbb{R}^{+}\right)$of the inequality (2.1), there exists a unique solution $v \in C^{1}\left(\mathcal{J}, \mathbb{R}^{+}\right)$of the considered problem (1.1)-(1.3), such that

$$
|u(t)-v(t)| \leq \varphi(\epsilon), t \in \mathcal{J} .
$$

Definition 2.7. [32] The FBVP (1.1)-(1.3) is said to be Ulam-Hyers-Rassias stable with respect to $\phi \in C\left(\mathcal{J}, \mathbb{R}^{+}\right)$, if there exists a non zero positive real number $\mathcal{K}_{\phi}$, such that for every $\epsilon>0$ and for every solution $u \in C^{1}(\mathcal{J}, \mathbb{R})$ of the inequality

$$
\left|D^{p} u(t)-f\left(t, u(t), D^{p} u(t)\right)\right| \leq \epsilon \phi(t), t \in \mathcal{J},
$$


there exists a unique solution $v \in C^{1}(\mathcal{J}, \mathbb{R})$ of the considered problem (1.1)-(1.3), such that

$$
|u(t)-v(t)| \leq \mathcal{K}_{\phi} \epsilon \phi(t), t \in \mathcal{J} .
$$

Definition 2.8. [32] The FBVP (1.1)-(1.3) is said to be generalized Ulam-Hyers-Rassias stable with respect to $\phi \in C(\mathcal{J}, \mathbb{R})$, if there exists $\mathcal{K}_{\phi} \in \mathbb{R}^{+}$, such that for every solution $u \in C^{1}(\mathcal{J}, \mathbb{R})$ of the inequality

$$
\left|D^{p} u(t)-f\left(t, u(t), D^{p} u(t)\right)\right| \leq \phi(t), t \in \mathcal{J},
$$

there exists a unique solution $v \in C^{1}(\mathcal{J}, \mathbb{R})$ of the considered problem (1.1)-(1.3), such that

$$
|u(t)-v(t)| \leq \mathcal{K}_{\phi} \phi(t), t \in \mathcal{J} .
$$

Remark 2.9. Clearly,

(i) Definition $2.5 \Longrightarrow$ Definition 2.6.

(ii) Definition $2.7 \Longrightarrow$ Definition 2.8 .

Remark 2.10. A function $u \in C^{1}(\mathcal{J}, \mathbb{R})$ is a solution of the inequality (2.1) if there exists a function $\psi \in C(\mathcal{J}, \mathbb{R})$ (dependent on $u$ ), such that

(I) $D^{p} u(t)=f\left(t, u(t), D^{p} u(t)\right)+\psi(t), t \in \mathcal{J}$;

$(I I)|\psi(t)| \leq \epsilon$, for all $t \in \mathcal{J}$.

Theorem 2.11. (Arzela-Ascoli's theorem $)[15]$ Let $\mathbf{B} \subset C(\mathcal{J}, \mathbb{R})$ is relatively compact and if

(1) $\mathbf{B}$ is uniformly bounded set such that there exists $\rho>0$ with

$$
\|f\|=\sup _{y \in \mathcal{J}}|f(y)|<\rho \text { for every } f \in \mathbf{B} .
$$

(2) $\mathbf{B}$ is equicontinuous set, i.e for every $\epsilon>0$, there exists $\delta>0$, such that for any

$$
y, \bar{y} \in \mathcal{J},|y-\bar{y}| \leq \delta \Rightarrow|f(y)-f(\bar{y})| \leq \epsilon \text {, for every } f \in \mathbf{B} .
$$

Theorem 2.12. (Banach fixed point theorem)[11] Let $\mathcal{B}$ be a non-empty closed subset of a Banach space $\mathcal{X}$. Then any contraction mapping $\mathcal{S}$ from $\mathcal{X}$ into itself has a unique fixed point.

Theorem 2.13. (Schaefer's fixed point theorem)[11] Let $X$ be a Banach space. Suppose that the operator $\mathcal{S}: \mathcal{X} \rightarrow \mathcal{X}$ be a continuous compact mapping (or completely continuous). Moreover, suppose

$$
\mathcal{B}=\{u \in X \mid u=\eta \mathcal{S} u, 0<\eta<1\}
$$

be a bounded set. Then $\mathcal{S}$ has at least one fixed point in $X$.

\section{Existence and uniqueness results}

In this portion, we are establish adequate conditions for the existence and uniqueness of solutions to the considered FBVP (1.1)-(1.3).

Theorem 3.1. Let $\theta \in C(\mathcal{J}, \mathbb{R})$, then the equivalent Fredholm integral equation of the following boundary value problem

$$
D^{p} u(t)=\theta(t), \quad p \in(1,2], t \in \mathcal{J},
$$

with boundary conditions

$$
\begin{aligned}
& D^{p-2} u\left(0^{+}\right)=\gamma D^{p-2} u\left(T^{-}\right), \\
& D^{p-1} u\left(0^{+}\right)=\beta D^{p-1} u\left(T^{-}\right),
\end{aligned}
$$

is given by

$$
u(t)=\int_{0}^{T} \mathbb{G}(t, \zeta) \theta(\zeta) d \zeta,
$$


where $\mathbb{G}(t, \zeta)$ is the Green's function, in the following form

$$
\mathbb{G}(t, \zeta)= \begin{cases}\frac{1}{\Gamma(p)}(t-\zeta)^{p-1}+\frac{\beta t^{p-1}}{(1-\beta) \Gamma(p)}+\frac{\gamma t^{p-2}[T-(1-\beta) \zeta]}{(1-\beta)(1-\gamma) \Gamma(p-1)}, & 0 \leq \zeta \leq t \leq T, \\ \frac{\beta t^{p-1}}{(1-\beta) \Gamma(p)}+\frac{\gamma t^{p-2}[T-(1-\beta) \zeta]}{(1-\beta)(1-\gamma) \Gamma(p-1)}, & 0 \leq t \leq \zeta \leq T .\end{cases}
$$

Proof. Let us consider a linear boundary value problem given by

$$
D^{p} u(t)=\theta(t), \quad p \in(1,2], t \in \mathcal{J} .
$$

By Lemma 2.3, we have

$$
u(t)=k_{1} t^{p-1}+k_{2} t^{p-2}+I^{p} \theta(t) .
$$

Using boundary condition (3.1), we get

$$
k_{2}=\frac{\gamma}{(1-\gamma) \Gamma(p-1)}\left[k_{1} \Gamma(p) T+\int_{0}^{T}(T-\zeta) \theta(\zeta) d \zeta\right] .
$$

And now by using boundary condition (3.2), we obtain

$$
k_{1}=\frac{\beta}{(1-\beta) \Gamma(p)} \int_{0}^{T} \theta(\zeta) d \zeta .
$$

Put the value of $k_{1}$ in (3.5), it becomes

$$
k_{2}=\frac{\gamma}{(1-\gamma) \Gamma(p-1)}\left[T \frac{\beta}{1-\beta} \int_{0}^{T} \theta(\zeta) d \zeta+\int_{0}^{T}(T-\zeta) \theta(\zeta) d \zeta\right] .
$$

Plugging the value of $k_{1}$ and $k_{2}$ in (3.4), we have

$$
\begin{aligned}
u(t)= & \frac{1}{\Gamma(p)} \int_{0}^{t}(t-\zeta)^{p-1} \theta(\zeta) d \zeta+\frac{\beta t^{p-1}}{(1-\beta) \Gamma(p)} \int_{0}^{T} \theta(\zeta) d \zeta \\
& +\frac{\gamma t^{p-2}}{(1-\beta)(1-\gamma) \Gamma(p-1)} \int_{0}^{T}[T-(1-\beta) \zeta] \theta(\zeta) d \zeta
\end{aligned}
$$

Thus,

$$
u(t)=\int_{0}^{T} \mathbb{G}(t, \zeta) \theta(\zeta) d \zeta,
$$

here $\mathbb{G}(t, \zeta)$ is the given Green's function.

Therefore in view of Theorem 3.1, the solution of FBVP (1.1)-(1.3) is equivalent to the integral equation given by

$$
u(t)=\int_{0}^{T} \mathbb{G}(t, \zeta) f\left(s, u(\zeta), D^{p} u(\zeta)\right) d \zeta, t \in \mathcal{J} .
$$

Here we point out that for $\beta, \gamma=-1$, the boundary conditions (1.2) and (1.3) reduce to the boundary conditions of anti-periodic type:

$$
D^{p-2} u(0)=-D^{p-2} u(T), \quad D^{p-1} u(0)=-D^{p-1} u(T) .
$$

In this case the Green's function (3.3), takes the form

$$
\mathbb{G}(t, \zeta)= \begin{cases}\frac{1}{\Gamma(p)}(t-\zeta)^{p-1}-\frac{t^{p-1}}{2 \Gamma(p)}+\frac{t^{p-2}(2 \zeta-T)}{4 \Gamma(p-1)}, & 0 \leq \zeta \leq t \leq T, \\ \frac{-t^{p-1}}{2 \Gamma(p)}+\frac{t^{p-2}(2 \zeta-T)}{4 \Gamma(p-1)}, & 0 \leq t \leq \zeta \leq T .\end{cases}
$$

Lemma 3.2. The Green's function $\mathbb{G}(t, \zeta)$, which is obtained in the Theorem 3.1, will satisfy the following properties:

$\left(\mathcal{A}_{1}\right) \mathbb{G}(t, \zeta) \geq 0$ for all $t, \zeta \in \mathcal{J}$;

$\left(\mathcal{A}_{2}\right) \mathbb{G}(t, \zeta)$ is continuous over $\mathcal{J} \times \mathfrak{J}$; 


$$
\left(\mathcal{A}_{3}\right) \max _{t \in \mathcal{J}} \int_{0}^{T}|\mathbb{G}(t, \zeta)| d s \leq\left(\frac{T^{2}}{\Gamma(p+1)}+\left|\frac{\beta T^{2}}{(1-\beta) \Gamma(p)}\right|+\left|\frac{\gamma(1+|\beta|) T^{2}}{2(1-\beta)(1-\gamma) \Gamma(p-1)}\right|\right) .
$$

Proof. It is very easy to prove $\left(\mathcal{A}_{1}\right)$ and $\left(\mathcal{A}_{2}\right)$, so we leave it.

$\left(\mathcal{A}_{3}\right)$ : Since the Green's function of the considered problem in the following form

$$
\begin{aligned}
\int_{0}^{T}|\mathbb{G}(t, \zeta)| d \zeta & =\mid \frac{1}{\Gamma(p)} \int_{0}^{t}(t-\zeta)^{p-1} d \zeta+\frac{\beta t^{p-1}}{(1-\beta) \Gamma(p)} \int_{0}^{T} d \zeta \\
& +\frac{\gamma t^{p-2}}{(1-\beta)(1-\gamma) \Gamma(p-1)} \int_{0}^{T}[T-(1-\beta) \zeta] d \zeta \mid
\end{aligned}
$$

so, we get

$$
\max _{t \in \mathcal{J}} \int_{0}^{T}|\mathbb{G}(t, \zeta)| \leq\left(\frac{T^{2}}{\Gamma(p+1)}+\left|\frac{\beta T^{2}}{(1-\beta) \Gamma(p)}\right|+\left|\frac{\gamma(1+|\beta|) T^{2}}{2(1-\beta)(1-\gamma) \Gamma(p-1)}\right|\right)
$$

Hence this is complete the proof of $\left(\mathcal{A}_{3}\right)$.

Let the space $X=C(\mathcal{J}, \mathbb{R})$ be a Banach space with the following defined norm

$$
\|u\|_{X}=\max _{t \in \mathcal{J}}\{|u(t)|: t \in \mathcal{J}\} .
$$

If $u$ is the solution of FBVP (1.1)-(1.3), then

$$
\begin{aligned}
u(t)= & \frac{1}{\Gamma(p)} \int_{0}^{t}(t-\zeta)^{p-1} f(\zeta, u(\zeta), y(\zeta)) d \zeta+\frac{\beta t^{p-1}}{(1-\beta) \Gamma(p)} \int_{0}^{T} f(\zeta, u(\zeta), y(\zeta)) d \zeta \\
& +\frac{\gamma t^{p-2}}{(1-\beta)(1-\gamma) \Gamma(p-1)} \int_{0}^{T}[T-(1-\beta) \zeta] f(\zeta, u(\zeta), y(\zeta)) d \zeta, t \in \mathcal{J}
\end{aligned}
$$

where

$$
y(t)=D^{p} u(t)
$$

Define an operator $\mathcal{S}: \mathcal{X} \rightarrow \mathcal{X}$ as

$$
\begin{aligned}
\mathcal{S} u(t)= & \frac{1}{\Gamma(p)} \int_{0}^{t}(t-\zeta)^{p-1} y(\zeta) d \zeta+\frac{\beta t^{p-1}}{(1-\beta) \Gamma(p)} \int_{0}^{T} y(\zeta) d \zeta \\
& +\frac{\gamma t^{p-2}}{(1-\beta)(1-\gamma) \Gamma(p-1)} \int_{0}^{T}[T-(1-\beta) \zeta] y(\zeta) d \zeta, t \in \mathcal{J},
\end{aligned}
$$

where $y \in C(\mathcal{J}, \mathbb{R})$, such that

$$
y(t)=f(t, u(t), y(t)) .
$$

For further analysis, the following hypothesis need to be hold.

$\left(\mathcal{H}_{1}\right) \quad f: \mathcal{J} \times \mathbb{R} \times \mathbb{R} \rightarrow \mathbb{R}$ is continuous;

$\left(\mathcal{H}_{2}\right)$ For $t \in \mathcal{J}$, there exist constants $0<\mathbb{L}<1$ and $\mathbb{K}>0$, such that

$$
|f(t, v, x)-f(t, \bar{v}, \bar{x})| \leq \mathbb{K}|v-\bar{v}|+\mathbb{L}|x-\bar{x}| \text { for any } v, \bar{v}, x, \bar{x} \in \mathbb{R}
$$

$\left(\mathcal{H}_{3}\right)$ For $t \in \mathcal{J}$, there exist $a, b, c \in C\left(\mathcal{J}, \mathbb{R}^{+}\right)$, such that

$$
|f(t, u(t), w(t))| \leq a(t)+b(t)|u(t)|+c(t)|w(t)| \text { for } u, w \in \mathbb{R},
$$

with $a^{*}=\sup _{t \in \mathcal{J}} a(t), b^{*}=\sup _{t \in \mathcal{J}} b(t)$ and $c^{*}=\sup _{t \in \mathcal{J}} c(t)<1$.

Theorem 3.3. Under the hypothesis $\left(\mathcal{H}_{1}\right)-\left(\mathcal{H}_{3}\right)$, the operator $\mathcal{S}$ is compact.

Proof. Consider the operator $\mathcal{S}$ defined in (3.9). We have to show that the operator $\mathcal{S}$ is compact. The proof of this theorem will be given in several steps. 
$\operatorname{Step}(\mathbf{1})$ : Let the operator $\mathcal{S}$ be continuous, suppose a sequence $\left\{u_{n}\right\}$ such that $u_{n} \rightarrow u$ in $X$, then for each $t \in \mathcal{J}$, we have

$$
\begin{aligned}
& \quad\left|\mathcal{S}\left(u_{n}\right)(t)-\mathcal{S}(u)(t)\right|= \\
& \mid \frac{1}{\Gamma(p)} \int_{0}^{t}(t-\zeta)^{p-1}\left(y_{n}(\zeta)-y(\zeta)\right) d \zeta+\frac{\beta t^{p-1}}{(1-\beta) \Gamma(p)} \int_{0}^{T}\left(y_{n}(\zeta)-y(\zeta)\right) d \zeta \\
& \quad+\frac{\gamma t^{p-2}}{(1-\beta)(1-\gamma) \Gamma(p-1)} \int_{0}^{T}[T-(1-\beta) \zeta]\left(y_{n}(\zeta)-y(\zeta)\right) d \zeta \mid \\
& \leq \frac{1}{\Gamma(p)} \int_{0}^{t}(t-\zeta)^{p-1}\left|y_{n}(\zeta)-y(\zeta)\right| d \zeta+\frac{\beta t^{p-1}}{(1-\beta) \Gamma(p)} \int_{0}^{T}\left|y_{n}(\zeta)-y(\zeta)\right| d \zeta \\
& \quad+\frac{\gamma t^{p-2}}{(1-\beta)(1-\gamma) \Gamma(p-1)} \int_{0}^{T}[T-(1-\beta) \zeta]\left|y_{n}(\zeta)-y(\zeta)\right| d \zeta,
\end{aligned}
$$

where $y_{n}, y \in C(\mathcal{J}, \mathbb{R})$ and

$$
\begin{gathered}
y_{n}=f\left(t, u_{n}(t), y_{n}(t)\right), \\
y=f(t, u(t), y(t)) .
\end{gathered}
$$

Now by $\left(\mathcal{H}_{2}\right)$, we have

$$
\begin{aligned}
\left|y_{n}(t)-y(t)\right| & =\mid f\left(t, u_{n}(t), y_{n}(t)-f(t, u(t), y(t)) \mid\right. \\
& \leq \mathbb{K}\left|u_{n}(t)-u(t)\right|+\mathbb{L}\left|y_{n}(t)-y(t)\right| .
\end{aligned}
$$

So, we get

$$
\left|y_{n}(t)-y(t)\right| \leq \frac{\mathbb{K}}{1-\mathbb{L}}\left|u_{n}(t)-u(t)\right| .
$$

Since we supposed that $u_{n} \rightarrow u$, then $y_{n} \rightarrow y$ as $n \rightarrow \infty$ for each $t \in \mathcal{J}$. So by Lebesgue Dominated Convergence Theorem [9], (3.10) implies that

$$
\left|\mathcal{S}\left(u_{n}\right)(t)-\mathcal{S}(u)(t)\right| \rightarrow 0 \quad \text { as } \quad n \rightarrow \infty,
$$

hence

$$
\left\|\mathcal{S}\left(u_{n}\right)-\mathcal{S}(u)\right\|_{x} \rightarrow 0 \quad \text { as } \quad n \rightarrow \infty .
$$

As a result, $\mathcal{S}$ is continuous.

$\operatorname{Step}(2)$ : Now we are to prove that the operator $\mathcal{S}$ is bounded in set $\mathcal{X}$. For this we just to show that for any $\xi^{*}>0$, there exist $\wp>0$, such that for each

$$
u \in \mathbf{E}^{*}=\left\{u \in \mathcal{X}:\|u\|_{X} \leq \xi^{*}\right\},
$$

then, we have

$$
\|\mathcal{S}(u)\|_{x} \leq \wp .
$$

Since from (3.9), for each $t \in \mathcal{J}$, we have

$$
\begin{aligned}
\mathcal{S} u(t)= & \frac{1}{\Gamma(p)} \int_{0}^{t}(t-\zeta)^{p-1} y(\zeta) d \zeta+\frac{\beta t^{p-1}}{(1-\beta) \Gamma(p)} \int_{0}^{T} y(\zeta) d \zeta \\
& +\frac{\gamma t^{p-2}}{(1-\beta)(1-\gamma) \Gamma(p-1)} \int_{0}^{T}[T-(1-\beta) \zeta] y(\zeta) d \zeta
\end{aligned}
$$

where $y \in C(\mathcal{J}, \mathbb{R})$, such that

$$
y(t)=f(t, u(t), y(t)) .
$$


Now by $\left(\mathcal{H}_{3}\right)$, we have

$$
\begin{aligned}
|y(t)| & =|f(t, u(t), y(t))| \\
& \leq a(t)+b(t)|u(t)|+c(t)|y(t)| \\
& \leq a(t)+b(t) \xi^{*}+c(t)|y(t)| \\
& \leq a^{*}+b^{*} \xi^{*}+c^{*}\|y\|_{x} .
\end{aligned}
$$

Therefore, we get

$$
\|y\|_{x} \leq \frac{a^{*}+b^{*} \xi^{*}}{1-c^{*}}=\mathcal{M}_{0} .
$$

In this way (3.11) becomes

$$
|\mathcal{S}(u)(t)| \leq \mathcal{M}_{0} T^{p}\left(\frac{1}{\Gamma(p+1)}+\left|\frac{\beta}{(1-\beta \Gamma(p)}\right|+\left|\frac{\gamma(1+|\beta|)}{2(1-\beta)(1-\gamma) \Gamma(p-1)}\right|\right),
$$

which implies that

$$
\|\mathcal{S}(u)\|_{x} \leq \mathcal{M}_{0} T^{2}\left(\frac{1}{\Gamma(p+1)}+\left|\frac{\beta}{(1-\beta) \Gamma(p)}\right|+\left|\frac{\gamma(1+|\beta|)}{2(1-\beta)(1-\gamma) \Gamma(p-1)}\right|\right)=\wp .
$$

Hence $\mathcal{S}\left(\mathbf{E}^{*}\right)$ is uniformly bounded.

$\operatorname{Step}(3)$ : Now we are to show that the operator $\mathcal{S}$ is equicontinuous in $\mathcal{X}$. For this let $t_{1}, t_{2} \in \mathcal{J}$ with $t_{1}>t_{2}$, since $\mathbf{E}^{*}$ a bounded set in $\mathcal{X}$, and let $u \in \mathbf{E}^{*}$. Then

$$
\begin{aligned}
& \left|\mathcal{S}(u)\left(t_{1}\right)-\mathcal{S}(u)\left(t_{2}\right)\right|= \\
& \mid \frac{1}{\Gamma(p)} \int_{0}^{t_{1}}\left[\left(t_{1}-\zeta\right)^{p-1}-\left(t_{2}-\zeta\right)^{p-1}\right] y(\zeta) d \zeta-\frac{1}{\Gamma(p)} \int_{t_{1}}^{t_{2}}\left(t_{2}-\zeta\right)^{p-1} y(\zeta) d \zeta \\
& +\frac{\beta\left(t_{1}^{p-1}-t_{2}^{p-1}\right)}{(1-\beta) \Gamma(p-1)} \int_{0}^{T} y(\zeta) d \zeta \mid
\end{aligned}
$$

by using (3.12), we get

$$
\left|\mathcal{S}(u)\left(t_{1}\right)-\mathcal{S}(u)\left(t_{2}\right)\right| \leq \mathcal{M}_{0}\left(\left|\frac{1}{\Gamma(p+1)}\left[2\left(t_{2}-t_{1}\right)^{p}-\left(t_{2}^{p}-t_{1}^{p}\right)\right]\right|+\left|\frac{\beta\left(t_{2}^{p-1}-t_{1}^{p-1}\right) T}{(1-\beta) \Gamma(p-1)}\right|\right) .
$$

As $t_{1} \rightarrow t_{2}$, then the right-hand side of the above inequality tends to zero. Hence $\mathcal{S}\left(\mathbf{E}^{*}\right)$ is equicontinuous. As a consequence of step (1) to (3) the operator $\mathcal{S}$ is completely continuous. Therefore in view of Arzela-Ascoli theorem, the operator $\mathcal{S}$ is compact.

Theorem 3.4. Let the hypothesis $\left(\mathcal{H}_{3}\right)$ holds and if $N<1$. Then FBVP (1.1)-(1.3) has at least one solution in $X$.

Proof. For the proof of this theorem, we are considering a set $\mathcal{B} \subset \mathcal{X}$, which is defined in the following form

$$
\mathcal{B}=\{u \in \mathcal{X}: u=\eta \mathcal{S} u, 0<\eta<1\} .
$$

We have to show that the set $\mathcal{B}$ is bounded. Let $u \in \mathcal{B}$, such that

$$
u(t)=\eta \mathfrak{S} u(t) \text {, where } \eta \in(0,1) .
$$

Then for each $t \in \mathcal{J}$, we have

$$
\begin{aligned}
|u(t)|= & \mid \eta\left(\frac{1}{\Gamma(p)} \int_{0}^{t}(t-\zeta)^{p-1} y(\zeta) d \zeta+\frac{\beta t^{p-1}}{(1-\beta) \Gamma(p)} \int_{0}^{T} y(\zeta) d \zeta\right. \\
& \left.+\frac{\gamma t^{p-2}}{(1-\beta)(1-\gamma) \Gamma(p-1)} \int_{0}^{T}[T-(1-\beta) \zeta] y(\zeta) d \zeta\right) \mid, \\
\leq & \frac{T^{p}}{\Gamma(p+1)}|y(\zeta)|+\left|\frac{\beta T^{p}}{(1-\beta) \Gamma(p)}\right||y(\zeta)|+\left|\frac{\gamma T^{p}(1+|\beta|)}{2(1-\beta)(1-\gamma) \Gamma(p-1)}\right| y(\zeta) \mid .
\end{aligned}
$$


Now by $\left(\mathcal{H}_{3}\right)$ for each $t \in \mathcal{J}$,

$$
\begin{aligned}
|y(t)| & =|f(t, u(t), y(t))| \\
& \leq a(t)+b(t)|u(t)|+c(t)|y(t)| \\
& \leq a^{*}+b^{*}|u(t)|+c^{*}|y(t)| .
\end{aligned}
$$

So, we get

$$
|y(t)| \leq \frac{1}{1-c^{*}}\left(a^{*}+b^{*}|u(t)|\right) .
$$

Plugging (3.14) in (3.13), so it becomes

$$
\begin{aligned}
|u(t)| \leq & \frac{T^{p}\left(\frac{1}{1-c^{*}}\left(a^{*}+b^{*}|u(t)|\right)\right)}{\Gamma(p+1)}+\frac{\beta T^{p}\left(\frac{1}{1-c^{*}}\left(a^{*}+b^{*}|u(t)|\right)\right)}{(1-\beta) \Gamma(p)} \\
& +\frac{\gamma T^{p}(1+\beta)\left(\frac{1}{1-c^{*}}\left(a^{*}+b^{*}|u(t)|\right)\right)}{2(1-\beta)(1-\gamma) \Gamma(p-1)} .
\end{aligned}
$$

Taking maximum on both sides, we get

$$
\begin{aligned}
\|u\|_{x} \leq & \frac{T^{2}\left(\frac{1}{1-c^{*}}\left(a^{*}+b^{*}\|u\|_{x}\right)\right)}{\Gamma(p+1)}+\frac{\beta T^{2}\left(\frac{1}{1-c^{*}}\left(a^{*}+b^{*}\|u\|_{x}\right)\right)}{(1-\beta) \Gamma(p)} \\
& +\frac{\gamma T^{2}(1+\beta)\left(\frac{1}{1-c^{*}}\left(a^{*}+b^{*}\|u\|_{x}\right)\right)}{2(1-\beta)(1-\gamma) \Gamma(p-1)} .
\end{aligned}
$$

For simplicity, let say

$$
W=\frac{T^{2} a^{*}}{\left(1-c^{*}\right) \Gamma(p+1)}+\frac{T^{2} a^{*} \beta}{(1-\beta)\left(1-c^{*}\right) \Gamma(p)}+\frac{T^{2} a^{*} \gamma(1+|\beta|)}{2(1-\beta)(1-\gamma)\left(1-c^{*}\right) \Gamma(p-1)}
$$

and

$$
N=\frac{T^{2} b^{*}}{\left(1-c^{*}\right) \Gamma(p+1)}+\frac{T^{2} b^{*} \beta}{(1-\beta)\left(1-c^{*}\right) \Gamma(p)}+\frac{T^{2} b^{*} \gamma(1+|\beta|)}{2(1-\beta)(1-\gamma)\left(1-c^{*}\right) \Gamma(p-1)} .
$$

So (3.15) becomes

$$
\|u\|_{x} \leq N\|u\|_{x}+W
$$

We get

$$
\|u\|_{x} \leq \frac{W}{1-N}
$$

This shows that the set $\mathcal{B}$ is bounded. So by Theorem 3.3 and Theorem 2.13 , we get that the operator $\mathcal{S}$ has at least one fixed point. Therefore, the considered FBVP (1.1)-(1.3) has at least one solution in $\mathcal{X}$.

Theorem 3.5. Suppose that the hypothesis $\left(\mathcal{H}_{1}\right)$ and $\left(\mathcal{H}_{2}\right)$ hold. Then the FBVP (1.1)(1.3) has a unique solution in $X$. If

$$
\frac{\mathbb{K} T^{2}}{1-\mathbb{L}}\left(\frac{1}{\Gamma(p+1)}+\left|\frac{\beta}{(1-\beta) \Gamma(p)}\right|+\left|\frac{\gamma(1+|\beta|)}{2(1-\beta)(1-\gamma) \Gamma(p-1)}\right|\right)<1 .
$$

Proof. Since the operator $\mathcal{S}: \mathcal{X} \rightarrow X$ defined in (3.9) is

$$
\begin{aligned}
\mathcal{S} u(t)= & \frac{1}{\Gamma(p)} \int_{0}^{t}(t-\zeta)^{p-1} y(\zeta) d \zeta+\frac{\beta t^{p-1}}{(1-\beta) \Gamma(p)} \int_{0}^{T} y(\zeta) d \zeta \\
& +\frac{\gamma t^{p-2}}{(1-\beta)(1-\gamma) \Gamma(p-1)} \int_{0}^{T}[T-(1-\beta) \zeta] y(\zeta) d \zeta, t \in \mathcal{J},
\end{aligned}
$$

where $y \in C(\mathcal{J}, \mathbb{R})$, such that

$$
y(t)=f(t, u(t), y(t)) .
$$


Here we shall use Banach contraction principle to prove the operator $\mathcal{S}$ has unique fixed point, which will be the unique solution of the FBVP (1.1)-(1.3).

Let $u, \bar{u} \in \mathcal{X}$ and for $t \in \mathcal{J}$, we have

$$
\begin{aligned}
& |\mathcal{S}(u)(t)-\mathcal{S}(\bar{u})(t)| \leq \\
& \frac{1}{\Gamma(p)} \int_{0}^{t}(t-\zeta)^{p-1}|y(\zeta)-g(\zeta)| d \zeta+\frac{\beta t^{p-1}}{(1-\beta) \Gamma(p)} \int_{0}^{T}|y(\zeta)-g(\zeta)| d \zeta \\
& +\frac{\gamma t^{p-2}}{(1-\beta)(1-\gamma) \Gamma(p-1)} \int_{0}^{T}[T-(1-\beta) \zeta]|y(\zeta)-g(\zeta)| d \zeta,
\end{aligned}
$$

where $y, g \in C(\mathcal{J}, \mathbb{R})$, such that

$$
y(t)=f(t, u(t), y(t))
$$

and

$$
g(t)=f(t, \bar{u}(t), g(t))
$$

Now by $\left(\mathcal{H}_{2}\right)$, we have

$$
\begin{aligned}
|y(t)-g(t)| & =|f(t, u(t), y(t))-f(t, \bar{u}(t), g(t))| \\
& \leq \mathbb{K}|u(t)-\bar{u}(t)|+\mathbb{L}|y(t)-g(t)| .
\end{aligned}
$$

Thus

$$
|y(t)-g(t)| \leq \frac{\mathbb{K}}{1-\mathbb{L}}|u(t)-\bar{u}(t)|
$$

So (??) becomes

$$
\begin{aligned}
& |\mathcal{S}(u)(t)-\mathcal{S}(\bar{u})(t)| \leq \\
& \frac{\mathbb{K}}{1-\mathbb{L}}\left(\frac{1}{\Gamma(p)} \int_{0}^{t}(t-\zeta)^{p-1}|u(\zeta)-\bar{u}(\zeta)| d \zeta+\frac{\beta t^{p-1}}{(1-\beta) \Gamma(p)} \int_{0}^{T}|u(\zeta)-\bar{u}(\zeta)| d \zeta\right. \\
& \left.+\frac{\gamma t^{p-2}}{(1-\beta)(1-\gamma) \Gamma(p-1)} \int_{0}^{T}[T-(1-\beta) \zeta]|u(\zeta)-\bar{u}(\zeta)| d \zeta\right) .
\end{aligned}
$$

Now taking maximum on both sides, we get

$\|\mathcal{S}(u)-\mathcal{S}(\bar{u})\|_{x} \leq \frac{\mathbb{K} T^{2}}{1-\mathbb{L}}\left(\frac{1}{\Gamma(p+1)}+\left|\frac{\beta}{(1-\beta) \Gamma(p)}\right|+\left|\frac{\gamma(1+|\beta|)}{2(1-\beta)(1-\gamma) \Gamma(p-1)}\right|\right)\|u-\bar{u}\|_{x}$.

Hence the operator $\mathcal{S}$ is a contraction. Thus, by Banach contraction principle, we get that $\mathcal{S}$ has a unique fixed point, which is a unique solution of the FBVP (1.1)-(1.3).

\section{Ulam stability analysis}

In this portion, we are developing some sufficient conditions under which the proposed FBVP (1.1)-(1.3) will satisfy the hypothesis of various kinds of Ulam stability.

Lemma 4.1. Let $1<p \leq 2$, if $z \in C^{1}(\mathcal{J}, \mathbb{R})$ is the solution of the inequality (2.1), then $z$ will be the solution of the following integral inequality

$$
|z(t)-m(t)| \leq\left(\frac{T^{p}}{\Gamma(p+1)}+\left|\frac{\beta T^{p}}{(1-\beta) \Gamma(p)}\right|+\left|\frac{\gamma(1+|\beta|) T^{p}}{2(1-\beta)(1-\gamma) \Gamma(p-1)}\right|\right) \epsilon .
$$

Proof. Let $z$ be the solution of inequality (2.1). So in view (I) of Remark (2.10), we have

$$
\left\{\begin{array}{l}
D^{p} z(t)=f\left(t, z(t), D^{p} z(t)\right)+\psi(t), t \in \mathcal{J}, \\
D^{p-2} z\left(0^{+}\right)=\gamma D^{p-2} z\left(T^{-}\right) \\
D^{p-1} z\left(0^{+}\right)=\beta D^{p-1} z\left(T^{-}\right) .
\end{array}\right.
$$


So, the solution of (4.1) will be in the following form

$$
\begin{aligned}
z(t)= & \frac{1}{\Gamma(p)} \int_{0}^{t}(t-\zeta)^{p-1} y(\zeta) d \zeta+\frac{\beta t^{p-1}}{(1-\beta) \Gamma(p)} \int_{0}^{T} y(\zeta) d \zeta \\
& +\frac{\gamma t^{p-2}}{(1-\beta)(1-\gamma) \Gamma(p-1)} \int_{0}^{T}[T-(1-\beta) \zeta] y(\zeta) d \zeta \\
& +\frac{1}{\Gamma(p)} \int_{0}^{t}(t-\zeta)^{p-1} \psi(\zeta) d \zeta+\frac{\beta t^{p-1}}{(1-\beta) \Gamma(p)} \int_{0}^{T} \psi(\zeta) d \zeta \\
& +\frac{\gamma t^{p-2}}{(1-\beta)(1-\gamma) \Gamma(p-1)} \int_{0}^{T}[T-(1-\beta) \zeta] \psi(\zeta) d \zeta
\end{aligned}
$$

For simplicity, let us denote the sum of terms free of $\psi$ by $m(t)$, we have

$$
\begin{aligned}
m(t)= & \frac{1}{\Gamma(p)} \int_{0}^{t}(t-\zeta)^{p-1} y(\zeta) d \zeta+\frac{\beta t^{p-1}}{(1-\beta) \Gamma(p)} \int_{0}^{T} y(\zeta) d \zeta \\
& +\frac{\gamma t^{p-2}}{(1-\beta)(1-\gamma) \Gamma(p-1)} \int_{0}^{T}[T-(1-\beta) \zeta] y(\zeta) d \zeta
\end{aligned}
$$

So from above, we have

$$
\begin{aligned}
|z(t)-m(t)| \leq & \frac{1}{\Gamma(p)} \int_{0}^{t}(t-\zeta)^{p-1}|\psi(\zeta)| d \zeta+\frac{\beta t^{p-1}}{(1-\beta) \Gamma(p)} \int_{0}^{T}|\psi(\zeta)| d \zeta \\
& +\frac{\gamma t^{p-2}}{(1-\beta)(1-\gamma) \Gamma(p-1)} \int_{0}^{T}|T-(1-\beta) \zeta \| \psi(\zeta)| d \zeta
\end{aligned}
$$

Hence by using (II) of Remark 2.10, we get the following required result

$$
|z(t)-m(t)| \leq\left(\frac{T^{p}}{\Gamma(p+1)}+\left|\frac{\beta T^{p}}{(1-\beta) \Gamma(p)}\right|+\left|\frac{\gamma(1+|\beta|) T^{p}}{2(1-\beta)(1-\gamma) \Gamma(p-1)}\right|\right) \epsilon
$$

Theorem 4.2. Let the hypothesis $\left(\mathcal{H}_{1}\right)$ and $\left(\mathcal{H}_{2}\right)$ hold along with the condition

$$
\left(\frac{\mathbb{K} T^{2}}{(1-\mathbb{L}) \Gamma(p+1)}+\left|\frac{\beta \mathbb{K} T^{2}}{(1-\mathbb{L})(1-\beta) \Gamma(p)}\right|+\left|\frac{\gamma(1+|\beta|) \mathbb{K} T^{2}}{2(1-\mathbb{L})(1-\beta)(1-\gamma) \Gamma(p-1)}\right|\right)<1 .
$$

Then the FBVP (1.1)-(1.3) will be Ulam-Hyers stable and consequently generalized UlamHyers stable.

Proof. Suppose $z \in C^{1}(\mathcal{J}, \mathbb{R})$ be any solution of the inequality (2.1) and $v$ be the unique solution of the considered FBVP (1.1)-(1.3), then we have

$$
\left\{\begin{array}{l}
D^{p} v(t)=f\left(t, v(t), D^{p} v(t)\right), p \in(1,2], t \in \mathcal{J}, \\
D^{p-2} v\left(0^{+}\right)=\gamma D^{p-2} v\left(T^{-}\right) \\
D^{p-1} v\left(0^{+}\right)=\beta D^{p-1} v\left(T^{-}\right) .
\end{array}\right.
$$

Now

$$
|z(t)-v(t)|=|z(t)-m(t)+m(t)-v(t)| \leq|z(t)-m(t)|+|m(t)-v(t)| .
$$


By using Lemma 4.1 in (4.2), we have

$$
\begin{aligned}
& |z(t)-v(t)| \leq \\
& \left(\frac{T^{p}}{\Gamma(p+1)}+\left|\frac{\beta T^{p}}{(1-\beta) \Gamma(p)}\right|+\left|\frac{\gamma(1+|\beta|) T^{p}}{2(1-\beta)(1-\gamma) \Gamma(p-1)}\right|\right) \epsilon \\
& +\mid \frac{1}{\Gamma(p)} \int_{0}^{t}(t-\zeta)^{p-1}\left(y_{z}(\zeta)-g_{v}(\zeta)\right) d \zeta+\frac{\beta t^{p-1}}{(1-\beta) \Gamma(p)} \int_{0}^{T}\left(y_{z}(\zeta)-g_{v}(\zeta)\right) d \zeta \\
& +\frac{\gamma t^{p-2}}{(1-\beta)(1-\gamma) \Gamma(p-1)} \int_{0}^{T}[T-(1-\beta) \zeta]\left(y_{z}(\zeta)-g_{v}(\zeta)\right) d \zeta \mid
\end{aligned}
$$

We get

$$
\begin{aligned}
& |z(t)-v(t)| \leq \\
& \left(\frac{T^{p}}{\Gamma(p+1)}+\left|\frac{\beta T^{p}}{(1-\beta) \Gamma(p)}\right|+\left|\frac{\gamma(1+|\beta|) T^{p}}{2(1-\beta)(1-\gamma) \Gamma(p-1)}\right|\right) \epsilon \\
& +\left(\frac{T^{p}}{\Gamma(p+1)}+\left|\frac{\beta T^{p}}{(1-\beta) \Gamma(p)}\right|+\left|\frac{\gamma(1+|\beta|) T^{p}}{2(1-\beta)(1-\gamma) \Gamma(p-1)}\right|\right)\left|y_{z}(\zeta)-g_{v}(\zeta)\right| .
\end{aligned}
$$

For $y_{z}, g_{v} \in C(\mathcal{J}, \mathbb{R})$, where

$$
y_{z}(t)=f\left(t, z(t), y_{z}(t)\right)
$$

and

Using $\left(\mathcal{H}_{2}\right)$, then we have

$$
g_{v}(t)=f\left(t, v(t), g_{v}(t)\right)
$$

$$
\begin{aligned}
\left|y_{z}(t)-g_{v}(t)\right| & =\left|f\left(t, z(t), y_{z}(t)\right)+f\left(t, v(t), g_{v}(t)\right)\right| \\
& \leq \mathbb{K}|z(t)-v(t)|+\mathbb{L}|y(t)-g(t)| .
\end{aligned}
$$

So we get

$$
\left|y_{z}(t)-g_{v}(t)\right| \leq \frac{\mathbb{K}}{1-\mathbb{L}}|z(t)-v(t)|
$$

Using (4.4) and for each $t \in \mathcal{J}$, (??) implies that

$$
\begin{aligned}
\|z-v\|_{x} \leq & \left(\frac{T^{2}}{\Gamma(p+1)}+\left|\frac{\beta T^{2}}{(1-\beta) \Gamma(p)}\right|+\left|\frac{\gamma(1+|\beta|) T^{2}}{2(1-\beta)(1-\gamma) \Gamma(p-1)}\right|\right) \epsilon \\
& +\left(\frac{\mathbb{K} T^{2}}{(1-\mathbb{L}) \Gamma(p+1)}+\left|\frac{\beta \mathbb{K} T^{2}}{(1-\mathbb{L})(1-\beta) \Gamma(p)}\right|\right. \\
& \left.+\left|\frac{\gamma(1+|\beta|) \mathbb{K} T^{2}}{2(1-\mathbb{L})(1-\beta)(1-\gamma) \Gamma(p-1)}\right|\right)\|z-v\|_{x} .
\end{aligned}
$$

After simplification, we get

$$
\|z-v\|_{x} \leq\left(\frac{\frac{T^{2}}{\Gamma(p+1)}+\left|\frac{\beta T^{2}}{(1-\beta) \Gamma(p)}\right|+\left|\frac{\gamma(1+|\beta|) T^{2}}{2(1-\beta)(1-\gamma) \Gamma(p-1)}\right|}{1-\left(\frac{\mathbb{K} T^{2}}{(1-\mathbb{L}) \Gamma(p+1)}+\left|\frac{\beta \mathbb{K} T^{2}}{(1-\mathbb{L})(1-\beta) \Gamma(p)}\right|+\left|\frac{\gamma(1+|\beta|) \mathbb{K} T^{2}}{2(1-\mathbb{L})(1-\beta)(1-\gamma) \Gamma(p-1)}\right|\right)}\right) \epsilon .
$$

Thus, we have

$$
|z(t)-v(t)| \leq \mathcal{K}_{0} \epsilon
$$

where

such that

$$
\mathcal{K}_{0}=\frac{\frac{T^{2}}{\Gamma(p+1)}+\left|\frac{\beta T^{2}}{(1-\beta) \Gamma(p)}\right|+\left|\frac{\gamma(1+|\beta|) T^{2}}{2(1-\beta)(1-\gamma) \Gamma(p-1)}\right|}{1-\left(\frac{\mathbb{K} T^{2}}{(1-\mathbb{L}) \Gamma(p+1)}+\left|\frac{\beta \mathbb{K} T^{2}}{(1-\mathbb{L})(1-\beta) \Gamma(p)}\right|+\left|\frac{\gamma(1+|\beta|) \mathbb{K} T^{2}}{2(1-\mathbb{L})(1-\beta)(1-\gamma) \Gamma(p-1)}\right|\right)},
$$

$$
\left(\frac{\mathbb{K} T^{2}}{(1-\mathbb{L}) \Gamma(p+1)}+\left|\frac{\beta \mathbb{K} T^{2}}{(1-\mathbb{L})(1-\beta) \Gamma(p)}\right|+\left|\frac{\gamma(1+|\beta|) \mathbb{K} T^{2}}{2(1-\mathbb{L})(1-\beta)(1-\gamma) \Gamma(p-1)}\right|\right)<1 .
$$


Thus, the FBVP (1.1)-(1.3) is Ulam-Hyers stable.

Now by putting $\varphi(\epsilon)=\mathcal{K}_{0} \epsilon, \varphi(0)=0$ yields that the FBVP (1.1)-(1.3) is generalized Ulam-Hyers stable. This is complete the proof.

$\left(\mathcal{H}_{4}\right)$ Suppose a function $\phi \in\left(\mathcal{J}, \mathbb{R}^{+}\right)$, which is increasing. Then there exists $\mu_{\phi}>0$, such that for each $t \in \mathcal{J}$, the following integral inequality

$$
I^{p} \phi(t) \leq \mu_{\phi} \phi(t)
$$

holds.

Lemma 4.3. Let the hypothesis $\left(\mathcal{H}_{4}\right)$ hold and suppose $z \in C^{1}(\mathcal{J}, \mathbb{R})$ is the solution of the inequality (2.2), then $z$ is a solution of the following integral inequality

$$
\begin{aligned}
& |z(t)-m(t)| \leq \\
& \left(\mu_{\phi}+\left|\frac{\beta T^{p-1}}{(1-\beta) \Gamma(p)}\right| \mu_{\phi}+\left|\frac{\gamma \beta T^{p-1}}{(1-\beta)(1-\gamma) \Gamma(p-1)}\right| \mu_{\phi}+\left|\frac{\gamma T^{p-2}}{(1-\gamma) \Gamma(p-1)}\right| \mu_{\phi}\right) \phi(t) \epsilon .
\end{aligned}
$$

Proof. Since from Lemma 4.1, we have

$$
\begin{aligned}
|z(t)-m(t)| \leq & \frac{1}{\Gamma(p)} \int_{0}^{t}(t-\zeta)^{p-1}|\psi(\zeta)| d \zeta+\frac{\beta t^{p-1}}{(1-\beta) \Gamma(p)} \int_{0}^{T}|\psi(\zeta)| d \zeta \\
& +\frac{\gamma t^{p-2}}{(1-\beta)(1-\gamma) \Gamma(p-1)} \int_{0}^{T}[T-(1-\beta) \zeta]|\psi(\zeta)| d \zeta .
\end{aligned}
$$

After using (II) of Remark 2.10 and $\left(\mathcal{H}_{4}\right)$, we obtain the following required inequality

$$
\begin{aligned}
& |z(t)-m(t)| \leq \\
& \left(\mu_{\phi}+\left|\frac{\beta T^{p-1}}{(1-\beta) \Gamma(p)}\right| \mu_{\phi}+\left|\frac{\gamma \beta T^{p-1}}{(1-\beta)(1-\gamma) \Gamma(p-1)}\right| \mu_{\phi}+\left|\frac{\gamma T^{p-2}}{(1-\gamma) \Gamma(p-1)}\right| \mu_{\phi}\right) \phi(t) \epsilon .
\end{aligned}
$$

Theorem 4.4. Let the hypothesis $\left(\mathcal{H}_{1}\right),\left(\mathcal{H}_{2}\right)$ and $\left(\mathcal{H}_{4}\right)$ hold. Then the FBVP (1.1)-(1.3) is said to be Ulam-Hyers-Rassias stable and generalized Ulam-Hyers-Rassias stable. If

$$
1-\left(\frac{\mathbb{K} T^{2}}{(1-\mathbb{L}) \Gamma(p+1)}+\left|\frac{\beta \mathbb{K} T^{2}}{(1-\mathbb{L})(1-\beta) \Gamma(p)}\right|+\left|\frac{\gamma(1+|\beta|) \mathbb{K} T^{2}}{2(1-\mathbb{L})(1-\beta)(1-\gamma) \Gamma(p-1)}\right|\right)>0 .
$$

Proof. Suppose $z \in C^{1}(\mathcal{J}, \mathbb{R})$ be any solution of the inequality (2.2) and let $v$ be the unique solution of the considered FBVP (1.1)-(1.3). Then for each $t \in \mathcal{J}$, we have

$$
|z(t)-v(t)|=|z(t)-m(t)+m(t)-v(t)| \leq|z(t)-m(t)|+|m(t)-v(t)| .
$$

Using $\left(\mathcal{H}_{2}\right)$ as a similar way like in Theorem 4.2 , we get

$$
\begin{aligned}
|m(t)-v(t)| \leq & \left(\frac{\mathbb{K} T^{p}}{(1-\mathbb{L}) \Gamma(p+1)}+\left|\frac{\beta \mathbb{K} T^{p}}{(1-\mathbb{L})(1-\beta) \Gamma(p)}\right|\right. \\
& \left.+\left|\frac{\gamma(1+|\beta|) \mathbb{K} T^{p}}{2(1-\mathbb{L})(1-\beta)(1-\gamma) \Gamma(p-1)}\right|\right)|z(\zeta)-v(\zeta)| .
\end{aligned}
$$

Now by Lemma 4.3 and by (4.7), (4.6) becomes

$$
\begin{aligned}
& \|z-v\| x \leq \\
& \left(\mu_{\phi}+\left|\frac{\beta T}{(1-\beta) \Gamma(p)}\right| \mu_{\phi}+\left|\frac{\gamma \beta T}{(1-\beta)(1-\gamma) \Gamma(p-1)}\right| \mu_{\phi}+\left|\frac{\gamma}{(1-\gamma) \Gamma(p-1)}\right| \mu_{\phi}\right) \phi(t) \epsilon \\
+ & \left(\frac{\mathbb{K} T^{2}}{(1-\mathbb{L}) \Gamma(p+1)}+\left|\frac{\beta \mathbb{K}^{2}}{(1-\mathbb{L})(1-\beta) \Gamma(p)}\right|+\left|\frac{\gamma(1+|\beta|) \mathbb{K} T^{2}}{2(1-\mathbb{L})(1-\beta)(1-\gamma) \Gamma(p-1)}\right|\right)\|z-v\|_{x} .
\end{aligned}
$$


Rearrange the terms, we get

$$
\|z-v\|_{X} \leq\left(\frac{\mu_{\phi}+\left|\frac{\beta T}{(1-\beta) \Gamma(p)}\right| \mu_{\phi}+\left|\frac{\gamma \beta T}{(1-\beta)(1-\gamma) \Gamma(p-1)}\right| \mu_{\phi}+\left|\frac{\gamma}{(1-\gamma) \Gamma(p-1)}\right| \mu_{\phi}}{1-\left(\frac{\mathbb{K} T^{2}}{(1-\mathbb{L}) \Gamma(p+1)}+\left|\frac{\beta \mathbb{K} T^{2}}{(1-\mathbb{L})(1-\beta) \Gamma(p)}\right|+\left|\frac{\gamma(1+|\beta|) \mathbb{K} T^{2}}{2(1-\mathbb{L})(1-\beta)(1-\gamma) \Gamma(p-1)}\right|\right)}\right) \phi(t) \epsilon .
$$

Thus, we have

$$
|z(t)-v(t)| \leq \mathcal{K}_{\phi} \phi(t) \epsilon,
$$

where

$$
\mathcal{K}_{\phi}=\frac{\mu_{\phi}+\left|\frac{\beta T}{(1-\beta) \Gamma(p)}\right| \mu_{\phi}+\left|\frac{\gamma \beta T}{(1-\beta)(1-\gamma) \Gamma(p-1)}\right| \mu_{\phi}+\left|\frac{\gamma}{(1-\gamma) \Gamma(p-1)}\right| \mu_{\phi}}{1-\left(\frac{\mathbb{K} T^{2}}{(1-\mathbb{L}) \Gamma(p+1)}+\left|\frac{\beta \mathbb{K} T^{2}}{(1-\mathbb{L})(1-\beta) \Gamma(p)}\right|+\left|\frac{\gamma(1+|\beta|) \mathbb{K} T^{2}}{2(1-\mathbb{L})(1-\beta)(1-\gamma) \Gamma(p-1)}\right|\right)},
$$

and

$$
1-\left(\frac{\mathbb{K} T^{2}}{(1-\mathbb{L}) \Gamma(p+1)}+\left|\frac{\beta \mathbb{K} T^{2}}{(1-\mathbb{L})(1-\beta) \Gamma(p)}\right|+\left|\frac{\gamma(1+|\beta|) \mathbb{K} T^{2}}{2(1-\mathbb{L})(1-\beta)(1-\gamma) \Gamma(p-1)}\right|\right)>0 .
$$

Hence, the FBVP (1.1)-(1.3) is Ulam-Hyer-Rassias stable.

Now, if we plug $\epsilon=1$ in (4.8), then by definition (2.8) the considered problem is generalized Ulam-Hyers-Rassias stable. This is complete the proof.

\section{Examples}

In this portion, we are illustrating the obtained results by couple of examples.

Example 5.1. Suppose the fractional order boundary value problem

$$
\left\{\begin{array}{l}
D^{\frac{5}{4}} u(t)=\frac{2+|u(t)|+\left|D^{\frac{5}{4}} u(t)\right|}{70 e^{t+12}\left(1+|u(t)|+\left|D^{\frac{5}{4}} u(t)\right|\right)}, t \in[0,1], \\
D^{\frac{-3}{4}} u\left(0^{+}\right)=\frac{1}{2} D^{\frac{-3}{4}} u\left(1^{-}\right), \\
D^{\frac{1}{4}} u\left(0^{+}\right)=-D^{\frac{1}{4}} u\left(1^{-}\right) .
\end{array}\right.
$$

Where

Set

$$
p=\frac{5}{4}, T=1, \gamma=\frac{1}{2} \text { and } \beta=-1
$$

$$
f(t, y, z)=\frac{(2+|y|+|z|)}{70 e^{t+12}(1+|y|+|z|)}, t \in[0,1], y, z \in \mathbb{R} .
$$

Clearly, the above function $f$ is jointly continuous.

Now for any $y, z, \bar{y}, \bar{z} \in \mathbb{R}$ and $t \in[0,1]$, we have

$$
|f(t, y, z)-f(t, \bar{y}, \bar{z})| \leq \frac{1}{70 e^{12}}(|y-\bar{y}|+|z-\bar{z}|) .
$$

Thus, $\left(\mathcal{H}_{2}\right)$ is satisfied with $\mathbb{K}=\mathbb{L}=\frac{1}{70 e^{12}}$.

Also, we have

$$
|f(t, y, z)| \leq \frac{1}{70 e^{t+12}}(2+|y|+|z|) .
$$

Thus, $\left(\mathcal{H}_{3}\right)$ satisfied with

$$
m(t)=\frac{1}{35 e^{t+12}}, b(t)=c(t)=\frac{1}{70 e^{t+12}},
$$

where $a^{*}=\frac{1}{35 e^{12}}$ and $b^{*}=c^{*}=\frac{1}{70 e^{12}}$.

Now, plug $T=1, \beta=-1, \gamma=\frac{1}{2}, p=\frac{5}{4}$ and $\mathbb{K}=\mathbb{L}=\frac{1}{70 e^{12}}$ in (3.16), we get

$$
\frac{1}{70 e^{12}-1}\left(\frac{1}{\Gamma\left(\frac{9}{4}\right)}+\frac{1}{2 \Gamma\left(\frac{5}{4}\right)}+\frac{1}{2 \Gamma\left(\frac{1}{4}\right)}\right) \approx 1.379946 \times 10^{-7}<1,
$$


thus condition (3.16) is satisfied. So from Theorem 3.5 the solution of the problem (5.1) is unique.

Furthermore, $\mathcal{K}_{0}>0$ with condition (4.5) is hold and

$$
\left(\frac{1}{\left(70 e^{12}-1\right) \Gamma\left(\frac{9}{4}\right)}+\frac{1}{2\left(70 e^{12}-1\right) \Gamma\left(\frac{5}{4}\right)}+\frac{1}{2\left(70 e^{12}-1\right) \Gamma\left(\frac{1}{4}\right)}\right) \approx 1.379946 \times 10^{-7}<1,
$$

so by Theorem 4.2, considered problem (5.1) is Ulam-Hyers stable and hence generalized Ulam-Hyers stable. Also by checking the condition of Theorem 4.4, it can be easily derived that the considered problem is Ulam-Hyers-Rassias stable and generalized Ulam-HyersRassias stable.

Example 5.2. Suppose the fractional order boundary value problem

$$
\left\{\begin{array}{l}
D^{\frac{3}{2}} u(t)=\frac{1}{90}(t \cos u(t)-u(t) \sin (t))+\frac{\left|D^{\frac{3}{2}} u(t)\right|}{45+\left|D^{\frac{3}{2}} u(t)\right|}, t \in[0,1], \\
D^{\frac{-1}{2}} u\left(0^{+}\right)=\frac{1}{2} D^{\frac{-1}{2}} u\left(1^{-}\right), \\
D^{\frac{1}{2}} u\left(0^{+}\right)=-D^{\frac{1}{2}} u\left(1^{-}\right) .
\end{array}\right.
$$

Where

$$
p=\frac{3}{2}, T=1, \gamma=\frac{1}{2} \text { and } \beta=-1
$$

Set

$$
f(t, y, z)=\frac{1}{90}(t \cos y-y \sin (t))+\frac{z}{45+z}, t \in[0,1], y, z \in \mathbb{R} .
$$

Clearly, the above function $f$ is jointly continuous.

Now for any $y, z, \bar{y}, \bar{z} \in \mathbb{R}$ and $t \in[0,1]$, we have

$$
\begin{aligned}
|f(t, y, z)-f(t, \bar{y}, \bar{z})| & \leq \frac{1}{90}|t||\cos y-\cos \bar{y}|+\frac{1}{90}|\sin (t)||y-\bar{y}|+\frac{45|z-\bar{z}|}{(45+z)(45-\bar{z})} \\
& \leq \frac{1}{90}|y-\bar{y}|+\frac{1}{90}|y-\bar{y}|+\frac{1}{45}|z-\bar{z}| \\
& \leq \frac{1}{45}|y-\bar{y}|+\frac{1}{45}|z-\bar{z}| \\
& \leq \frac{1}{45}(|y-\bar{y}|+|z-\bar{z}|) .
\end{aligned}
$$

Thus, $\left(\mathcal{H}_{2}\right)$ is satisfied with $\mathbb{K}=\mathbb{L}=\frac{1}{45}$.

Also, plug $T=1, \gamma=\frac{1}{2}, \beta=-1$ and $\mathbb{K}=\mathbb{L}=\frac{1}{45}$ in (3.16), we get

$$
\frac{1}{44}\left(\frac{4}{3 \sqrt{\pi}}+\frac{1}{\sqrt{\pi}}+\frac{1}{2 \sqrt{\pi}}\right) \approx 0.036323<1,
$$

thus condition (3.16) is satisfied. So from Theorem 3.5 the solution of the problem (5.2) is unique.

Furthermore, $\mathcal{K}_{0}>0$ with condition (4.5) is hold and

$$
\left(\frac{1}{33 \sqrt{\pi}}+\frac{1}{44 \sqrt{\pi}}+\frac{1}{88 \sqrt{\pi}}\right) \approx 0.036323<1,
$$

so by Theorem 4.2, considered problem (5.2) is Ulam-Hyers stable and hence generalized Ulam-Hyers stable. Also by checking the condition of Theorem 4.4, it can be easily seen that the considered problem (5.2) is Ulam-Hyers-Rassias stable and generalized UlamHyers-Rassias stable. 


\section{Conclusion}

We have effectively settled the existence and uniqueness conditions for a class of implicit FBVP (1.1)-(1.3) by using Schaefer's fixed point theorem, Banach contraction principle and Arzela-Ascoli theorem. Further, we additionally built up some proper conditions for different kinds of Ulam stability. Also, we illustrated our main results by providing couple of intrusting examples.

Acknowledgment. We are very thankful to the anonymous referee for his/her careful reading and suggestions which improved the quality of this paper.

\section{References}

[1] B. Ahmad and J.J. Nieto, Riemann-Laiouville fractional differential equations with fractional boundary conditions, Fixed Point Theor, 13 (2), 329-336, 2012.

[2] B. Ahmad and S. Sivasundaram, On four-point nonlocal boundary value problems of nonlinear integro-differential equations of fractional order, Appl. Math. Comput. 217, 480-487, 2010.

[3] N. Ahmad, Z. Ali, K. Shah, A. Zada and G. Ur Rahman, Analysis of implicit type nonlinear dynamical problem of impulsive fractional differential equations, Complexity, 2018, 1-15, 2018.

[4] Z. Ali, A. Zada and K. Shah, Existence and stability analysis of three point boundary value problem, Int. J. Appl. Comput. Math. 2017, DOI:10.1007/s40819-017-0375-8.

[5] G.A. Anastassiou, On right fractional calculus, Chaos Solitons Fractals, 42 (1), 365$376,2009$.

[6] D. Baleanu, Z.B. Güvenc and J.A.T. Machado, New Trends in Nanotechnology and Fractional Calculus Applications, Springer, New York, 2010.

[7] M. Benchohra and S. Bouriah, Existence and Stability Results for Nonlinear Boundary Value Problem for Implicit Differential Equations of Fractional Order, Moroccan J. Pure Appl. Anal. 1 (1), 22-37, 2015.

[8] M. Benchohra, S. Hamani and S.K. Ntouyas, Boundary value problems for differential equations with fractional order and nonlocal conditions, Nonlinear Anal. 71, 23912396, 2009.

[9] A. Browder, Mathematical Analysis: An Introduction, New York, Springer-Verlag, 1996.

[10] M. El-Shahed and J.J. Nieto, Nontrivial solutions for a nonlinear multi-point boundary value problem of fractional order, Comput. Math. Appl. 59, 3438-3443, 2010.

[11] A. Granas and J. Dugundji, Fixed Point Theory, Springer-Verlag, New York, 2003.

[12] E. Hiffer (ed.), Application of Fractional Calculus in Physics, Word Scientific, Singapore, 2000 .

[13] D.H. Hyers, On the stability of the linear functional equation, Proc. Natl. Acad. Sci. U.S.A, 27 (4), 222-224, 1941.

[14] D.H. Hyers, G. Isac and T.M. Rassias, Stability of Functional Equations in Several Variables, Birkhäiuser, Boston, 1998.

[15] J.K. Hale and S.M.V. Lunel, Introduction to Functional Differential Equations, in: Applied Mathematicals Sciences series, Springer-Verlag, New York, 99, 1993.

[16] R.W. Ibrahim, Generalized Ulam-Hyers stability for fractional differential equations, Int. J. Math. 23 (5), 9 pages, 2012.

[17] S.M. Jung, Hyers-Ulam stability of linear differential equations of first order, Appl. Math. Lett. 19, 854-858, 2006.

[18] R.A. Khan and K. Shah, K. Existence and uniqueness of solutions to fractional order multi-point boundary value problems, Commun. Appl. Anal. 19, 515-526, 2015. 
[19] A.A. Kilbas, H.M. Srivasta and J.J. Trujilllo, Theory and Application of Fractional Differential Equations, North-Holland Mathematics Studies, 24, North-Holand, Amsterdam, 2006.

[20] Y.H. Lee and K.W. Jun, A Generalization of the Hyers-Ulam-Rassias stability of Pexider equation, J. Math. Anal. Appl. 246, 627-638, 2000.

[21] T. Li and A. Zada, Connections between Hyers-Ulam stability and uniform exponential stability of discrete evolution families of bounded linear operators over Banach spaces, Adv. Difference Equ. 2016 (1), 1-8.

[22] T. Li, A. Zada and S. Faisal, Hyers-Ulam stability of nth order linear differential equations, J. Nonlinear Sci. Appl. 9, 2070-2075, 2016.

[23] J.T. Machado, V. Kiryakova and F. Mainardi, Recent history of fractional calculus, Commun. Nonlin. Sci. Numer. Simul. 16 (3), 1140-1153, 2011.

[24] R. Metzler and K. Joseph, Boundary value problems for fractional diffusion equations, Phys. A, 278 (1), 107-125, 2000.

[25] M. Obloza, Hyers stability of the linear differential equation, Rocznik Nauk-Dydakt. Prace Mat. 13, 259-270, 1993.

[26] K.B. Oldham, Fractional differential equations in electrochemistry, Adv. Eng. Softw. 41, 9-12, 2010.

[27] M.D. Ortigueira, Fractional Calculus for Scientists and Engineers, Lecture Notes in Electrical Engineering, 84, Springer, Dordrecht, 2011.

[28] I. Podlubny, Fractional Differential Equations, Academic Press, San Diego, 1999.

[29] T.M. Rassias, On the stability of the linear mapping in Banach spaces, Proc. Amer. Math. Soc. 72 (2), 297-300, 1978.

[30] T.M. Rassias, On the stability of functional equations and a problem of Ulam, Acta. Appl. Math. 62, 23-130, 2000.

[31] F.A. Rihan, Numerical Modeling of Fractional-Order Biological Systems, Abstr. Appl. Anal. 2013, 11 pages, 2013.

[32] I.A. Rus, Ulam stabilities of ordinary differential equations in a Banach space, Carpathian J. Math. 26, 103-107, 2010.

[33] J. Sabatier, O.P. Agrawal and J.A.T. Machado, Advances in fractional calculus, Dordrecht, Springer, 2007.

[34] K. Shah and R.A. Khan, Existence and uniqueness of positive solutions to a coupled system of nonlinear fractional order differential equations with anti periodic boundary conditions, Differ. Equ. Appl. 7 (2), 245-262, 2015.

[35] K. Shah, N. Ali and R.A. Khan, Existence of positive solution to a class of fractional differential equations with three point boundary conditions, Math. Sci. Lett. 5 (3), 291-296, 2016.

[36] K. Shah,H. Khalil and R.A. Khan, Investigation of positive solution to a coupled system of impulsive boundary value problems for nonlinear fractional order differential equations, Chaos Solitons Fractals, 77, 240-246, 2015.

[37] K. Shah, H. Khalil and R.A. Khan, Upper and lower solutions to a coupled system of nonlinear fractional differential equations, Prog. Fract. Differ. Appl. 1 (1), 1-8, 2016.

[38] X. Su and L. Liu, Existence of solution for boundary value problem of nonlinear fractional differential equation, Appl. Math. 22 (3), 291-298, 2007.

[39] S. Tang, A. Zada, S. Faisal, M.M.A. El-Sheikh and T. Li, Stability of higher order nonlinear impulsive differential equations, J. Nonlinear Sci. Appl. 9, 4713-4721, 2016.

[40] V.E. Tarasov, Fractional Dynamics: Application of Fractional Calculus to Dynamics of particles, Fields and Media, Springer, Heidelberg, Higher Education Press, Beijing, 2010.

[41] S.M. Ulam, A Collection of the Mathematical Problems, Interscience, New York, 1960. 
[42] B.M. Vintagre, I. Podlybni, A. Hernandez and V. Feliu, Some approximations of fractional order operators used in control theory and applications, Fract. Calc. Appl. Anal. 3 (3), 231-248, 2000.

[43] J. Wang, L. Lv and W. Zhou, Ulam stability and data dependence for fractional differential equations with Caputo derivative, Electron. J. Qual. Theory Differ. Equ. 63, 1-10, 2011.

[44] J.R. Wang, Y. Zhou and M. Fečkan, Nonlinear impulsive problems for fractional differential equations and Ulam stability, Comput. Math. Appl. 64 (10), 3389-3405, 2012.

[45] B. Xu, J. Brzdek and W. Zhang, Fixed point results and the Hyers-Ulam stability of linear equations of higher orders, Pacific J. Math. 273, 483-498, 2015.

[46] A. Zada, S. Faisal and Y. Li, On the Hyers-Ulam Stability of First Order Impulsive Delay Differential Equations, J. Func. Spac. 2016, 6 pages, 2016.

[47] A. Zada, O. Shah and R. Shah, Hyers-Ulam stability of non-autonomous systems in terms of boundedness of Cauchy problems, Appl. Math. Comput. 271, 512-518, 2015. 